\title{
C11orf95-RELA Fusion Protein Expression
}

National Cancer Institute

\section{Source}

National Cancer Institute. C110rf95-RELA Fusion Protein Expression. NCI Thesaurus.

Code C129352.

Expression of a fusion protein that results from the fusion of RELA and C11 orf95 genes.

It is present in the majority of supratentorial ependymomas in children. 